Rev. salud pública. 10 (3):452-461, 2008

\title{
Aluminio en el Tracto Respiratorio Bajo de Residentes de la Ciudad de México
}

\author{
Aluminium in the lower respiratory tract of people living \\ in México City \\ Adelaida R.I. Manquián-Tejos ${ }^{1}$, Luis R. Tovar-Gálvez ${ }^{1}$ y Gabriel Yáñez-Canal² \\ 1 Centro Interdisciplinario de Investigaciones y Estudios sobre Medio Ambiente y Desarrollo \\ (CIIEMAD). Instituto Politécnico Nacional (IPN). México. amanquiante yahoo.es \\ 2 Universidad Industrial de Santander, Colombia \\ Recibido 6 Diciembre 2007/Enviado para Modificación 8 Junio 2008/Aceptado 11 Junio 2008
}

\section{RESUMEN}

Objetivo Se investigó la presencia de aluminio $(A /)$ y de patologías respiratorias en el tracto respiratorio bajo de personas que habían residido en Ciudad de México por un tiempo mínimo de dos años.

Métodos Se obtuvo 250 muestras de tejido respiratorio (lóbulos pulmonares, nódulo linfático peribronquial, bronquios e hilio), durante la autopsia médico legal de 36 individuos. Para la cuantificación de Al se utilizó plasma de inducción acoplado (ICP OES) a la espectrofotometría de absorción atómica previo a lo cual las muestras fueron sometidas a un proceso de secado, trituración y digestión.

Resultados Se identificaron 13 diferentes patologías, solo tres de ellas: enfisema pulmonar, bronquitis y antracosis, se correlacionan con la presencia de $\mathrm{Al}$, elemento distribuido en concentraciones muy variables (rango:2,7 a 836,1 microgramos de $A$ l por gramo de tejido seco (ìg Al/g ts). En lóbulos, bronquios e hilio la cantidad de $A$ l encontrada fue mucho menor que la encontrada en nódulos peribronquiales, siendo la diferencia estadísticamente significativa. El análisis multivariado por conglomerados mostró que la muestra estaba conformada por tres clases de individuos, agrupados de acuerdo a la cantidad y distribución del $A$ l en el tracto respiratorio bajo, a la edad, tiempo de residencia en la ciudad de México y presencia de patologías.

Conclusión Se postula que el $A$ / encontrado en el tracto respiratorio bajo de residentes de ciudad de México proviene del aire; la cantidad y el patrón de distribución dependen del tiempo y del lugar de residencia y al depositarse puede provocar enfermedades respiratorias.

Palabras Clave: Contaminación del aire, material particulado, toxicidad, aluminio (fuente: DeCS, BIREME).

\section{ABSTRACT}

Objective Investigating the presence of aluminium $(A /)$ and respiratory pathologies in the lower respiratory tract of people who had lived in Mexico City for a minimum of two years. 
Methods 250 respiratory tissue samples were obtained from pulmonary lobes, lymph nodes, bronchial and hilum regions during 36 individuals' autopsies. Inductively coupled plasma optical emission spectrometry (ICP-OES) was used for quantifying $\mathrm{Al}$; the samples has been previously dried, ground and digested.

Results 13 different pathologies were identified but only three of them (pulmonary emphysema, bronchitis and anthracosis) were correlated with the presence of $A l$, an element being distributed in very variable concentrations (range: 2,7 to 836,1 micrograms of $A$ / per gram of dry tissue (ì g Al/g ts)). The amount of $A /$ found in lobes, bronchial and hilum regions was much smaller than that found in lymph nodes; such difference was statistically significant. Multivariate analysis by conglomerates revealed that the sample consisted of three classes of individuals, grouped according to the amount and distribution of $A$ / in the lower respiratory tract, age, time spent living in Mexico City and the presence of pathologies.

Conclusions The $A /$ found in the lower respiratory tract of residents of Mexico City would thus seem to have come from the air. The amount of $A l$ and its distribution pattern depended on the time and place of residence and can lead to respiratory illness.

Key Words: Air pollution, air pollutants, particulate, adverse effect, aluminum (source: MeSH, NLM).

$\mathrm{S}$ e ha demostrado que las partículas atmosféricas inducen incremento de la mortalidad $(1,2)$ y morbilidad $(2,3)$ en la población expuesta por causas tanto respiratorias (2-5) como cardiovasculares $(2,6,7)$ y del sistema nervioso central $(2,8)$. Los estudios experimentales muestran que los contaminantes del aire, en especial las partículas finas $\left(\mathrm{PM}_{10}\right)$ y ultrafinas $(10 \sim 100$ $\mathrm{nm})$ penetran en los alvéolos pulmonares y pueden causar reacciones inflamatorias, $(5,9)$, depositarse en la región toráxica (10) y vía sanguínea distribuirse a otros órganos tales como el corazón, $(2,5-7,9,11,12)$, e incluso al sistema nervioso central (8). Se postula que los metales transportados en la superficie de las partículas podrían ser algunos de los elementos responsables de tales efectos nocivos $(2,9,13)$.

En ciudad de México, tanto la presencia como la cantidad de metales encontrados en las partículas del aire varía de acuerdo con la zona geográfica, $(13,14)$. Entre los metales reportados se destaca la presencia de $\mathrm{Al}, \mathrm{Cu}, \mathrm{Fe}$, Ti, $\mathrm{Cr}, \mathrm{Mn}, \mathrm{Zn}, \mathrm{Mo}, \mathrm{V}, \mathrm{Ni}, \mathrm{Pb},(13,14)$. García (14), reporta que el Al es el metal

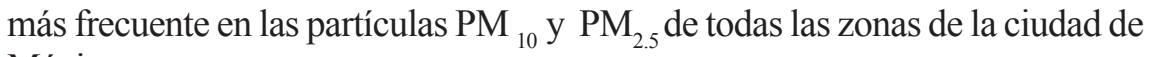
México.

Estos antecedentes motivaron la realización de un estudio químico y patológico, cuyo propósito fue evaluar en muestras de tejido respiratorio, de los cinco lóbulos del pulmón, del nódulo peribronquial y de los bronquios e hilio, la presencia y cantidad de metales y el posible daño asociado a su presencia. Se 
identificaron 17 metales, de los cuales es llamativo la presencia de $\mathrm{Al}$ en las muestras de tejido analizadas por varias razones. En primer lugar por su alta concentración, en segundo lugar porque se ha informado que es uno de los metales más frecuentemente encontrados en las partículas del aire de la ciudad de México (14) y, en tercer lugar, porque se sabe que el $A l$ no cumple con ninguna función biológica, por el contrario la inhalación de polvo de $A l$ puede causar patologías muy severas a nivel respiratorio $(4,15,16)$ y neurológico $(16,17)$.

\section{MÉTODOS}

En los años 2002, 2003 y 2004, se realizó un estudio de tipo exploratorio. Se obtuvieron 250 muestras de tejido respiratorio provenientes de cada lóbulo pulmonar, del nódulo linfático peribronquial, de bronquios y de la zona del hilio, durante la autopsia médico-legal de 36 individuos ( 24 hombres y 12 mujeres), que habían residido en la ciudad de México por un período superior a 2 años durante los cuales estuvieron expuestos a la contaminación atmosférica. Se excluyeron del estudio los individuos que habían sido fumadores, los expuestos laboralmente a metales y las embarazadas. Las muestras fueron obtenidas en el Servicio Médico Forense del DF (SEMEFO), previa autorización ético-legal otorgada por Honorable Consejo de la Judicatura.

Durante la obtención de las muestras, y con el objeto de evitar contaminación, se utilizó un bisturí de vidrio y cada muestra ( $15 \mathrm{~g}$ tejido húmedo) fue divida en dos: muestra A y muestra B. La muestra A fue preparada para estudio químico y la muestra B para estudio patológico.

Se indagó, además, sobre los antecedentes sociales, biológicos y de exposición en la ficha médico legal y a través de una entrevista personal a un familiar o conocido del fallecido.

Método de preparación de las muestras y análisis químico analítico. Las muestras sufrieron un proceso de preparación previa a la cuantificación de los elementos en espectrofotometría de absorción atómica.

A. Secado. Las muestras fueron secadas en un horno de convección forzada regulado a una temperatura de $90^{\circ}$ y durante un tiempo promedio de 24 horas. Durante este procedimiento se perdieron 28 de las 250 muestras.

B. Trituración del tejido. Durante este procedimiento se utilizaron herramientas de material inerte (sobres de papel albanene, mortero de loza, émbolo de plástico, émbolo de vidrio). 
C. Homogeneizado y toma de muestras para digestión. Se utilizó la técnica de cuarteo.

D. Digestión de las muestras. Para tal efecto se utilizó un horno microondas marca Perkin Elmer, manufacturado por Anton Paar (Graz Austria) y modelo Paar Physica. En cada digestión se preparó una muestra blanco conteniendo sólo los ácidos.

Análisis químico. Para cuantificar el $\mathrm{Al}$ se utilizó plasma de inducción acoplado (ICP-OES) a la espectrofotometría de absorción atómica. Previamente se midieron los límites de detección del instrumento, del método y el porcentaje de recuperación del elemento en estudio con el objeto de validar los resultados. Esta información se muestra en la Tabla 1.

Tabla 1. Límites de detección y porcentaje de recuperación del Aluminio en espectrofotometría de absorción atómica ICP-OES

\begin{tabular}{cccc} 
Límite detección instrumento ( ) & Límite detección método $(0)$ & $\%$ recuperación \\
\hline 0.1 & 0.3 & 37,75
\end{tabular}

$\left({ }^{a, b}\right)=\mu g / L$

Análisis Estadístico De acuerdo a las hipotesis: 1. El tejido pulmonar de personas que han vivido en la ciudad de México por más de 2 años contiene $A l$, el cual se distribuye de manera heterogénea en los diferentes segmentos del tracto respitarorio y se concentra en mayor proporción en el nódulo peribronquial. 2. La presencia de $\mathrm{Al}$ en el tejido respiratorio se asocia a daño y 3. Existen grupos de personas más vulnerables a acumular metales en el tejido pulmonar, el análisis estadístico contempló tres etapas: i) análisis exploratorio de los datos, ii) Test de distribución normal y prueba de hipótesis (se eligió el estadístico $t$ ); iii). Análisis de varianza (ANOVA) de un factor, previo a lo cual se retiraron los valores atípicos; el método utilizado es el recomendado por Moore (18); iii). Análisis multivariado (conglomerados). Los cálculos y los gráficos se realizaron en los paquetes SPSS 7.5, SPAD 4.5, SAS y Excel.

\section{RESULTADOS}

La muestra quedó conformada por 36 individuos (24 hombres y 12 mujeres), con una edad promedio de 44,7 años (rango 3 a 82 años) que habían residido en la ciudad de México un tiempo promedio de 23,6 años (rango 2 a 82 años).

El estudio patológico identificó 13 patologías respiratorias. De ellas solo el enfisema pulmonar, bronquitis y antracosis se asocian con la presencia de $\mathrm{Al}$ en el tracto respiratorio. 
La presencia de $\mathrm{Al}$ en el tracto respiratorio bajo es estadísticamente significativa ( $\mathrm{p}<0.001$ ). El rango de los valores es $2,7 \mu \mathrm{g} \mathrm{Al} / \mathrm{g}$ ts, valor mínimo, y $836,1 \mu \mathrm{g} \mathrm{Al} /$ $\mathrm{g}$ ts, valor máximo, las concentraciones muestran grandes variaciones individuales (CV entre 35,2 y 257,4\%), intersegmentos (CV 95,7\%) e intrasegmentos (CV entre 63,79 y $533,5 \%$ ).

Las mayor concentración de $A l$ se observó en el nódulo peribronquial. En lóbulos, bronquios e hilio la cantidad de $A l$ muestra valores similares (Tabla 2).

Tabla 2. Cantidad de $A$ l expresado en microgramos por gramo de tejido seco $(\mu \mathrm{g} / \mathrm{g}$ ts) en los diferentes segmentos respiratorios

\begin{tabular}{lllccc}
\hline \multicolumn{1}{c}{ Variable } & & $\mathrm{N}$ & Mediana & Mínimo & Máximo \\
\hline Lóbulo Superior Derecho & (LSD) & 31 & 38,5 & 6,8 & 177,4 \\
Lóbulo Medio Derecho & (LMD) & 32 & 45,5 & 8,1 & 152,5 \\
Lóbulo Inferior Derecho & (LID) & 33 & 40,0 & 2,7 & 185,7 \\
Lóbulo Superior Izquierdo & (LSI) & 33 & 42,4 & 5,6 & 166,2 \\
Lóbulo Inferior Izquierdo & (LII) & 33 & 37,0 & 2,7 & 166,9 \\
Nódulo Peribronquial & (NOD) & 33 & 160,1 & 13,4 & 836,1 \\
Bronquio & (BRO) & 15 & 42,8 & 9,6 & 118,2 \\
Zona del Hilio & (HIL) & 12 & 56,8 & 14,0 & 135,3 \\
\hline
\end{tabular}

Los valores de $\mathrm{Al}$ en lóbulos y nódulo se distribuyen de manera asimétrica a derecha y no siguen una distribución normal (test Anderson-Darling $\mathrm{p}<0.005$ ) a diferencia del comportamiento de los valores de las variables bronquio e hilio en los cuales las cantidades de $A l$ se distribuyen de manera más o menos simétrica y con distribución normal,(test Anderson-Darling $\mathrm{p}>0.2500$ ). Como se observa en la Figura 1 sólo la variable nódulo tiene un comportamiento diferente y esta diferencia es estadísticamente significativa (ANOVA factor F 20,27 p<0.000).

La cantidad de $\mathrm{Al}$ encontrada se relaciona con el género y la edad de los casos. En la Figura 2 se muestra esta relación, donde se puede observar que a mayor edad, mayor es la concentración de aluminio en el tracto respiratorio bajo en especial en el nódulo peri bronquial. Las mujeres mayores acumulan mayor cantidad de $\mathrm{Al}$ que los hombres mayores, en cambio en el grupo de menor edad son los hombres los que concentran una mayor cantidad de Al.

El análisis de conglomerados (cluster) clasificó la muestra en tres clases o grupos. Las variables o características consideradas fueron: la cantidad de $\mathrm{Al}$ acumulado en el tracto respiratorio bajo; la edad; variables de exposición ambiental tales como el tiempo y el lugar de residencia en la ciudad de México; tiempo de permanencia en ambiente intramuros; tiempo de permanencia en ambiente extramuros; horas de permanencia en el sitio laboral; y variables biológicas tales como estado nutricional y presencia de patologías. 
Figura 1. Comparación entre variables según la distribución de los valores de Al

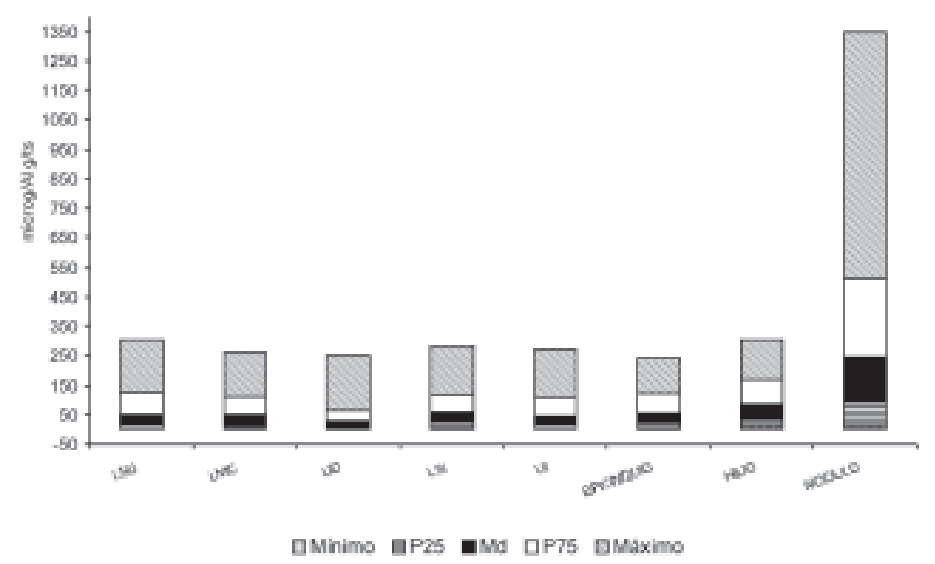

Figura 2. Concentración de Aluminio en nódulos peribronquiales según género y grupo de edad

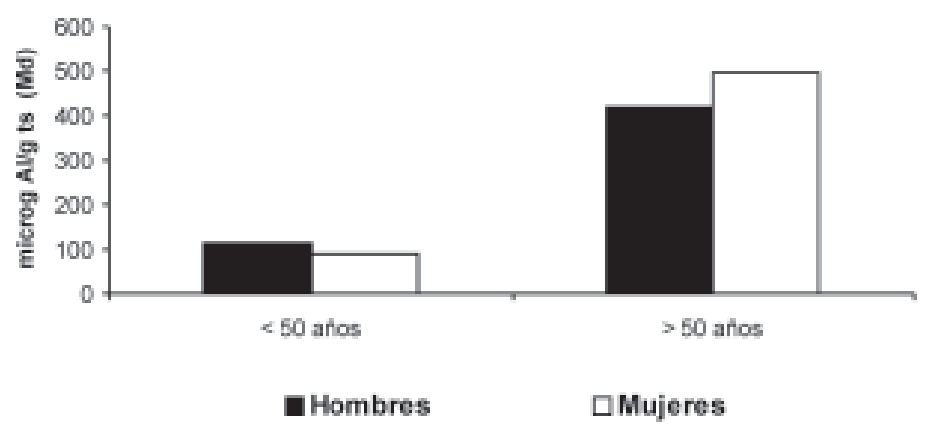

La primera clase agrupó los individuos de menor edad de la muestra (30 y menos años), con las concentraciones más bajas de $A l$, residentes de la zona sur de la ciudad, y las medias de clase de las variables de exposición ambiental inferiores a las medias generales, ausencia de patologías respiratorias y sobrepeso.

La segunda clase y tercera clase agruparon los casos de mayor edad de la muestra. La segunda clase se caracteriza por las concentraciones más altas de $\mathrm{Al}$ en lóbulos pulmonares, residentes de la zona centro de la ciudad por 30 años, con diagnóstico de enfisema pulmonar, estado nutricional normal y medias de las variables de exposición ambiental superiores a las medias generales. 
La tercera clase caracterizada por las concentraciones más elevadas de $A l$ en nódulos peribronquiales, residentes de la zona norte, estado nutricional bajo peso, diagnóstico de antracosis, antecedente de enfermedad cardiovascular $\mathrm{y}$, a diferencia de las dos primeras clases, las únicas variables de exposición que la caracterizan son tiempo de residencia y tiempo de permanencia en el lugar de trabajo; ambas variables muestran medias superiores a las medias generales.

\section{DISCUSIÓN}

El presente estudio reporta que las personas que han residido en la ciudad de México por más de dos años acumulan en el tracto respiratorio bajo, en especial en el nódulo peribronquial, $\mathrm{Al}$ en cantidades muy variables cuyos valores se distribuyen asimétricamente (a derecha) y de manera no normal en lóbulos y nódulo (test $\mathrm{A}-\mathrm{Darling} \mathrm{p}<0.005$ ); este comportamiento, ha sido descrito para metales no esenciales para la vida humana, los que al no cumplir con ninguna función biológica se comportan como contaminantes y sus niveles son controlados externamente, de tal manera que su patrón de distribución puede ser similar al de los niveles ambientales, (19) a diferencia del comportamiento de los elementos esenciales que al estar sujetos a un control interno muestran una distribución normal $(19,20)$.

Las investigaciones muestran que el nivel de toxicidad del $\mathrm{Al}$ puede ser muy bajo (21) y puede provocar enfermedades respiratorias $(4,16,21,22)$, neurológicas $(15,17,23)$ y recientemente se ha reportado su efecto cancerígeno $(24,25)$.

Los efectos respiratorios se han observado principalmente en trabajadores expuestos $(4,5,26,27)$ y estudios experimentales indican que la presencia de Al se correlaciona con proceso inflamatorio pulmonar $(22,28)$.

En este estudio se identificaron 13 diferentes patologías respiratorias, sin embargo, sólo tres de ellas se asociaron con la presencia de Al: enfisema pulmonar, (con las mayores concentraciones de $\mathrm{Al}$ en los lóbulos pulmonares); antracosis (con mayores concentraciones de $A l$ en nódulos peribronquiales); y bronquitis (con mayores concentraciones de $A l$ en bronquios).

El mecanismo de acción del $A l$ no ha sido del todo aclarado $(16,23)$. Un estudio experimental reciente (21) muestra que el $A l$ (óxido de $A l$ ) puede en muy bajas concentraciones disminuir la viabilidad de los macrófagos 
alveolares y entorpecer su función fagocítica, después de un período de 24 horas de exposición. Este mecanismo podría explicar, en primer lugar, la alta concentración de $\mathrm{Al}$ encontrada en nódulos peribronquiales (Md 160.1 $\mu \mathrm{gAl} / \mathrm{g}$ ts) comparada con la concentración encontrada en lóbulos (Md entre 37.0 y $45.5 \mu \mathrm{g} \mathrm{Al} / \mathrm{g}$ ts), bronquios (Md $42.8 \mu \mathrm{g} \mathrm{Al} / \mathrm{g}$ ts) e hilio (Md. $56.8 \mu \mathrm{g}$ $\mathrm{Al} / \mathrm{g}$ ts). En segundo lugar, la gran variabilidad de la distribución del $\mathrm{Al}$ tanto a nivel individual (CV entre 35,2 y $257,4 \%$ ), como colectivo para todos los segmentos respiratorios (CV 95,7\%) y para cada segmento en particular en todos los individuos (CV entre 63,79 y 533,5\%).

Las concentraciones de $A l$ en los nódulos peribronquiales están asociadas con el lugar de residencia: los casos con las concentraciones más altas de $\mathrm{Al}$ habían residido en la zona norte de la ciudad de México, reconocida como la zona industrial y donde se ha reportado que las partículas $\left(\mathrm{PM}_{10}\right)$ contienen una mayor concentración de $\mathrm{Al}$ comparadas con las partículas de otras zonas de la ciudad (14).

Con relación al género, el análisis univariado mostró que las mujeres mayores acumulan mayor cantidad de $\mathrm{Al}$ en los nódulos, comparado con los hombres de edades similares. Este resultado llama la atención ya que se ha reportado que las mujeres expuestas a la contaminación del aire tienen más riesgo que los hombres de morir y de presentar enfermedades cardiovasculares $(7,12)$.

De acuerdo con los resultados, el factor nutricional podría jugar un papel importante en el patrón de exposición personal, ya que a pesar de contar sólo con una aproximación diagnóstica del estado nutricional, observamos que el grupo con mayor cantidad de $A l$ en los nódulos peribronquiales tenía bajo peso.

El estudio por la misma forma en que se llevó a cabo, presenta algunas limitantes que dificultan inferir los resultados para las poblaciones de las cuales provenían los individuos estudiados. Además del pequeño tamaño muestral y de la imposibilidad de realizar una selección aleatoria, se tuvo que recurrir a fuentes secundarias (ficha médico legal, familiares o amigos) para recolectar la información de las variables biológicas y sociales. Adicionalmente, se presenta la imposibilidad de comparar los resultados con otros similares, debido a la falta de consenso de los laboratorios sobre los métodos de cuantificación y los estándares a utilizar (29). 
En conclusión: Los resultados sugieren que el $\mathrm{Al}$ encontrado en el tracto respiratorio bajo de residentes de ciudad de México: 1. Puede provenir del aire; 2. Que la cantidad de $\mathrm{Al}$ acumulado en el tracto respiratorio bajo es directamente proporcional al tiempo de exposición; 3. Que el patrón de distribución del Al depende de la zona de residencia; y 4. Que el $\mathrm{Al}$ al depositarse en el tracto respiratorio puede provocar enfermedades respiratorias probablemente porque es capaz de disminuir la viabilidad de los macrófagos alveolares y entorpecer su función fagocítica (21) •

Agradecimientos. Al Programa Institucional de Formación de Investigadores (PIFI) del IPN, por la beca y el financiamiento del estudio. Al Honorable Consejo de la Judicatura por otorgar el permiso para la obtención de muestras de tejido. Al Servicio Médico Forense del D.F. (SEMEFO) por las facilidades otorgadas en la etapa de muestreo. A la Dra. Elena Piña jefa del laboratorio de patología del SEMEFO por el aporte en el análisis de las muestras. A Lorena Campos y Ciro Marquéz Herrera, guías del análisis químico. Al Doctor Alvaro Osornio por sus aportes.

\section{REFERENCIAS}

1. O'Nelly M, Loomis D, Borja V, Gold D, Hertz I, Castillejos M. Do association between airborne particles and daily mortality in Mexico City differ by measurement method region, or modeling strategy?. J Expo Anal Environ Epidemiol 2004;14 (6): 429 - 39.

2. AHA (Scientific Statement). Brook R, Franklin B, Cascio W, Hong Y, Howard G, Lipsett M, Luepker R, Mittleman M, Samet J, Smith S, Tager I. Air pollution and cardiovascular diseases. A Statement for health care professionals from the expert panel on population and prevention science of the American Heart Association. Circulation 2004; 109: 2655-71.

3. Seaton A, MacNee W, Donaldson K, Godden D. Particulate air pollution and acute health effects. Lancet 1995; 345: 176-78.

4. Kelleher P, Pacheco K, Newman L. Inorganic dust pneumonias: The metal related parenchymal disorders. Environ Health Perspect 2000; 108 (Suppl 4): 685 - 96.

5. Monteiller C, Tran L, MacNee W, Faux S, Jones A, Miller B, Donaldson K. The pro-inflammatory effects of low-toxicity low-solubility particles, nanoparticles and fine particles, on epithelial cells in vitro: the role of surface area. Occup Environ Med. 2007 Sep; 64(9): 609-15.

6. Nemmar A, Hoet P, Vanquickenborne B, Dinsdale D, Thomeer M, Hoylaerts M, Vanbilloen H, Mortelmans L, Nemery B. Passage of inhaled particles into the blood circulation in humans. Circulation 2002; 105: $411-14$

7. Dockery DW, Stone PH. Cardiovascular risks from fine particulate air pollution. N Engl J Med. 2007;356(5): 511-3.

8. Kaiser J. Manganese: Ahigh Octane Dispute. Science 2003; 300: 926 - 28.

9. Mossman BT, Borm PJ, Castranova V, Costa DL, Donaldson K, Kleeberger SR. Mechanisms of action of inhaled fibers, particles and nanoparticles in lung and cardiovascular diseases.Part Fibre Toxicol. 2007; May 30:4:4.

10. Semmler-Behnke M, Takenaka S, Fertsch S, WenkA, Seitz J, Mayer P, Oberdörster G, Kreyling WG. Efficient elimination of inhaled nanoparticles from the alveolar region: evidence for 
interstitial uptake and subsequent reentrainment onto airways epithelium. Environ Health Perspect. 2007 May;115(5): 728-33.

11. Mills NL, Törnqvist H, Robinson SD, Gonzalez MC, Söderberg S, Sandström T, Blomberg A, Newby DE, Donaldson K. Air pollution and atherothrombosis. Inhal Toxicol. 2007;19 Suppl 1:81-9.

12. Miller KA, Siscovick DS, Sheppard L, Sheppard K, Sullivan JH, Anderson GL, Kaufman JD.. Long-term exposure to air pollution and incidence of cardiovascular events in women. N Engl J Med 2007;356: 447-458.

13. Bonner J. Rice A, Lindroos P, O'Brien P, Dreher K, Rosas I, Alfaro E, Osornio A. Induction of the lung myofibroblast PDGF receptor system by urban ambient particles from Mexico city. Respir Cell Mol Biol 1998; 19: 672-80.

14. García A, Icaza G, Angeles F, Blanco S, Gómez J, Delgadillo M, Mandujano F, Fentanes O, Segovia E, Salgado P, García H, Sepúlveda J, León E. Proyecto de monitoreo ambiental, microambiental y de exposición personal a partículas menores a diez micrómetros (PM10) y menores a 2.5 micrómetros (PM2.5) en la Zona Metropolitana de la Ciudad de México (ZMCM). Consejo de Estudios para la Restauración y Valoración Ambiental (CONSERVA), GDF; 2000.

15. Buchta M, Kiesswetter E, Otto A, Schaller K, Seeber A, Hilla W, Windorfer K, Stork J, Kuhlmann A, Gefeller O, Letzel S. Longitudinal study examining the neurotoxicity of occupational exposure to aluminium-containing welding fumes. Int Arch Occup Environ Health 2003; 76(7): 539-48.

16. Suwalsky M, Norris B, Villena F, Cuevas F, Sotomayor P, Zatta P. Aluminum fluoride affects the structure and functions of cell membranes. Food Chem Toxicol 2004; 42(6): 925-33.

17. Fattoretti P, Bertoni-Freddari C, Balietti M, Giorgetti B, Solazzi M, Zatta P. Chronic aluminum administration to old rats results in increased levels of brain metal ions and enlarged hippocampal mossy fibers. Ann N Y Acad Sci 2004; 1019: 44-7.

18. Moore D. Estadística Aplicada. $1^{\text {a }}$ Edición en castellano. Antoni Bosch; 1995.

19. Liebscher K, Smith H. Essential and nonessential trace elements. Arch Environ Health 1968; 17:881-90.

20. Thompson K, Orvig C. Boon and bane of metal ions in medicine. Science 2003; 300: 936-39.

21. Wagner AJ, Bleckmann CA, Murdock RC, Schrand AM, Schlager JJ, Hussain SM. Cellular interaction of different forms of aluminum nanoparticles in rat alveolar macrophages. $J$ Phys Chem B. 2007;111(25):7353-9.

22. Ha ${ }^{3}$ atek T, Trzcinka-Ochocka M, Matczak W, Grucha ${ }^{3}$ a J. Serum Clara cell protein as an indicator of pulmonary impairment in occupational exposure at aluminum foundry. Int $\mathrm{J}$ Occup Med Environ Health. 2006;19(4): 211-23.

23. Zatta P, Taylor A, Zambenedetti P, Milacic R, dell'Antone P. Aluminum inhibits the lysosomal proton pump from rat liver. Life Sci 2000; 66(23): 2261-6.

24. Spinelli JJ, Demers PA, Le ND, Friesen MD, Lorenzi MF, Fang R, Gallagher RP. Cancer risk in aluminum reduction plant workers (Canada). Cancer Causes Control. 2006 Sep;17(7): 939-48.

25. Exley C, Charles LM, Barr L, Martin C, PolwartA, Darbre PD. Aluminium in human breast tissue. J Inorg Biochem. 2007 Sep;101(9):1344-6.

26. Chan-Yeung M, Wong R, MacLean L, Tan F, Schulzer M, Enarson D, Martin A, Dennis R, Grzybowski S. Epidemiologic health study of workers in an aluminum smelter in British Columbia. Effects on the respiratory system. Am Rev Respir Dis 1983; 127: 465-469.

27. San L, Usysal H, Gokbel H, Bediz C, Sayal A. Pulmonary function of workers in the aluminum industry. Am J Ind Med 1998; 33 (3): 305-7.

28. Clarke R, Coull B, Reinisch U, Catalano P, Killingsworth C, Koutrakis P, Kavouras I, Gazula G, Murthy K, Lawrence J, Lovett E, Wolfson M, Verrier R, Godleski J. Inhaled concentrated ambient particles are associated with hematologic and bronchoalveolar lavage changes in canines. Environ Health Perspect 2000;108:1179-87.

29. Lovell M, Ehmann W, Markesbery W, Melethil S, Swyt C, Zatta P. Standardization in biological analyses of aluminum: what are the needs?. J Toxicol Environ Health 1996; 48(6): 63748. 